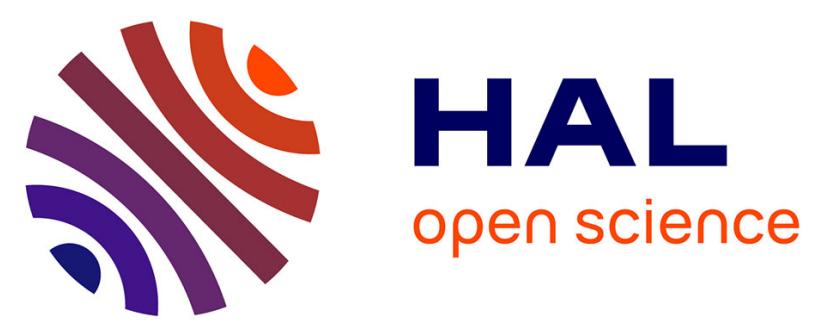

\title{
Assessment of Aspergillus Fumigatus Burden in Lungs of Intratracheally-Challenged Turkeys (Meleagris Gallopavo) by Quantitative PCR, Galactomannan Enzyme Immunoassay, and Quantitative Culture
}

Elise Melloul, Simon Thierry, Benoit Durand, Nathalie Cordonnier-Lefort, Guillaume Desoubeaux, Jacques Chandenier, Christophe Bostvironnois, Françoise Botterel, René Chermette, Jacques Guillot, et al.

\section{- To cite this version:}

Elise Melloul, Simon Thierry, Benoit Durand, Nathalie Cordonnier-Lefort, Guillaume Desoubeaux, et al.. Assessment of Aspergillus Fumigatus Burden in Lungs of Intratracheally-Challenged Turkeys (Meleagris Gallopavo) by Quantitative PCR, Galactomannan Enzyme Immunoassay, and Quantitative Culture. Comparative Immunology, Microbiology and Infectious Diseases, 2014, 37 (5-6), 10.1016/j.cimid.2014.07.005 . hal-02443804

\author{
HAL Id: hal-02443804 \\ https://hal.science/hal-02443804
}

Submitted on 22 Jan 2020

HAL is a multi-disciplinary open access archive for the deposit and dissemination of scientific research documents, whether they are published or not. The documents may come from teaching and research institutions in France or abroad, or from public or private research centers.
L'archive ouverte pluridisciplinaire HAL, est destinée au dépôt et à la diffusion de documents scientifiques de niveau recherche, publiés ou non, émanant des établissements d'enseignement et de recherche français ou étrangers, des laboratoires publics ou privés. 


\author{
Elise Melloul $^{1,5}$, Simon Thierry ${ }^{1}$, Benoit Durand ${ }^{2}$, Nathalie Cordonnier ${ }^{3}$, Jacques \\ Chandenier $^{4}$, Christophe Bostvironnois ${ }^{5}$, René Chermette ${ }^{1}$, Jacques Guillot ${ }^{1}$, Pascal \\ Arné $^{1 *}$ \\ 1 Research group ENVA, UPEC Dynamyc, Ecole Nationale Vétérinaire d'Alfort, UPE, Maisons-Alfort, \\ France \\ 2 Epidemiology, ANSES, LSA, Maisons-Alfort, France \\ 3 Pathology Department, Ecole Nationale Vétérinaire d'Alfort, Maisons-Alfort, France \\ 4 Parasitology-Mycology-Tropical Medicine, CHRU, Tours, France \\ 5 Lilly France, Elanco, Neuilly-sur-Seine, France \\ *Corresponding author. Mailing address: \\ 7, avenue du Général de Gaulle, 94704 Maisons-Alfort Cedex, France \\ parne@ vet-alfort.fr \\ +33143967073 \\ Short Title: Aspergillus pulmonary burden in an avian model
}


34

35

\section{7}

\begin{abstract}
- (less than 150 words)
Aspergillus fumigatus remains a major respiratory pathogen in birds, but diagnosis is still difficult. We challenged different groups of few-days-old turkeys via intratracheal aerosolisation of increasing concentrations $\left(10^{5}\right.$ up to $\left.10^{8}\right)$ of conidia using a MicroSprayer ${ }^{\circledR}$ device. The fungal burden was assessed by real-time PCR, galactomannan dosage, CFU counting and histopathological evaluation in order to provide a comparison of these results within each inoculum groups. A significant mortality occurring in the first 96 hours after inoculation was only observed with the highest inoculum dose. Culture counts, GM index and qPCR results on one hand and inoculum size on the other hand appeared to be clearly correlated. The mean fungal burden detected by qPCR was $1.3 \log _{10}$ units higher than mean values determined by CFU measurement. The new model and the markers will be used for the evaluation of the efficacy of antifungal treatments that could be applied in poultry farms.
\end{abstract}

\title{
$1 \quad$ Keywords (Up to 10)
}

52 Turkey, lung, aspergillosis, Aspergillus fumigatus, model, real-time PCR, galactomannan, 3 culture 


\section{Introduction}

Aspergillosis is the most severe fungal disease in birds involving thermophilic mold species belonging to the genus Aspergillus, among which A. fumigatus is by far the most prevalent causative agent. In poultry production and more specifically in turkeys' fast-growing or breeding flocks, this opportunistic infection induces severe economic losses. Generally confined to the lower respiratory tract in acute cases affecting young birds, lesions may extend to many other organs via secondary haematogenous dissemination or contiguous infection. The small diameter of conidia allow them, once inhaled, to be deposited directly deep within the respiratory system particularly in posterior air sacs. Therefore, primary colonization occurs in an optimal environment for germination and mycelium growth of Aspergillus molds. Considering the physiological specific aspects of their highly efficient respiratory tract and the lack of efficient treatments on declared aspergillosis, the development of avian models is absolutely necessary to acquire a comprehensive knowledge of the physiopathology of a disease which may be responsible for high rates of morbidity and mortality, poor performances and subsequent economic losses in poultry farms [1,2]. Numerous vertebrate models of this disease have been successively developed to study various aspects of the fungal development in the host, the subsequent immune response and to test antifungal drug efficacy. In contrast to rodents or rabbits experimental models, immune modulation is not a necessary prerequisite in avian challenge models [1]. Aspergillosis is readily induced in healthy birds by inhalation of aerosolized conidia in turkeys [3] or chickens [4], intravenous challenge in turkeys [5], intratracheal route in starlings [6], Japanese quails [7, 8, 9], rock pigeons and gyr-falcon hybrids [10] or by transcutaneous inoculation into air sacs in turkeys [11] or racing pigeons [12]. Besides mortality or clinical signs, the monitoring of host-pathogen interactions following experimental challenge in birds rely on gross lesions and histopathological evidences [13], 
completed with either the detection of markers of the fungal extension [1] or the quantification of the inflammatory response [7]. Since many years now, non-culture based methods either used alone or in combination have been targeted to Aspergillus DNA by realtime PCR or cell-wall antigens in different tissues or fluids to establish a rapid diagnosis of invasive aspergillosis in humans [14] or to monitor experimental disease in several rodents models $[15,16,17,18,19]$. Dosages of galactomannan or $1,3-\beta$-D-glucans have also been tested in different bird species suffering aspergillosis [20], in turkeys following experimental infection [21] and recently on field cases affecting commercial broiler chickens and turkeys [22].

Our goal was to validate an accurate model of acute aspergillosis in intratracheallychallenged turkeys. Non-culture-based methods and quantitative culture were compared to assess the fungal burden in lungs. In further investigations this original model may be used to test antifungal treatments in poultry.

\section{Materials and methods}

2.1. Aspergillus fumigatus strain, mycological cultures and preparation of the inoculum Aspergillus fumigatus strain CBS 144.89 was used for all experiments. This strain was initially isolated from a human patient with invasive aspergillosis in France and obtained from the Centraalbureau voor Schimmelculture (CBS), Utrecht, The Netherlands. All mycological cultures were done on malt agar plates supplemented with chloramphenicol (50 $\mathrm{mg} / \mathrm{L}$ ) and incubated at $37^{\circ} \mathrm{C}$ till 10 days depending on the fungal growth and species. To prepare the inoculum, the strain was grown for 10 days at $37^{\circ} \mathrm{C}$. Conidia were subsequently harvested by flooding the plates with sterile phosphate-buffered saline (PBS) containing 
$0.01 \%(\mathrm{vol} / \mathrm{vol})$ Tween 20 (PBST). The conidia were concentrated by centrifugation (at $3500 \mathrm{~g}$ for $30 \mathrm{~min}$ ) and counted using a Malassez counting chamber.

\subsection{Animals and housing conditions}

Conventional male turkeys (Le Sayec, Caudan, France) of 1-day-old with an average weight of 65 to $70 \mathrm{~g}$ were used. Groups of approximately 20 birds were housed in minimum $2 \mathrm{~m}^{2}$ cages on wood chips litter, in the biosafety 3 level sector of the animal facility of the veterinary college of Alfort, France. Animals had permanent access to fresh commercial poultry starter (Versela-Laga) and water. Photoperiod as ambient temperature was regulated and two infrared lamps provided additional heat during all the experiment.

\subsection{Environmental contamination assessment}

The fungal contamination of the environment was assessed before and during the experiments by regular sampling of conidia by sedimentation on two opened plates placed in each cage for $30 \mathrm{~min}$. In parallel, $1 \mathrm{~g}$ of litter and feedstuff were sampled and mixed in 45 mL PBST from which $100 \mu \mathrm{l}$ was seeded on agar plates.

\subsection{Nebulizer system and intratracheal challenge}

Two sets of experimental infections were conducted in the same conditions. For the first experiment, 36 seven-days-old turkeys were inoculated (day 0) with different concentrations of A. fumigatus (12 birds per inoculum): $10^{5}, 10^{6}$ or $10^{7}$ conidia per bird (groups named Inoc5, Inoc6 and Inoc7, respectively). Nine turkeys were inoculated with PBST as negative control. In the second experiment, 12 five-days-old turkeys were inoculated with $10^{8}$ conidia (Inoc8) and 7 turkeys were inoculated with PBST. Two birds of each group were 
anesthetized immediately after inoculation in order to check the contamination level of lungs.

Turkeys were anesthetized by inhalation of $5 \%$ isoflurane (Aerrane; Baxter) in oxygen and inoculated with $100 \mu \mathrm{L}$ of $A$. fumigatus suspension (or PBST) using a stainless steel 19 gauge atomizer (Microsprayer IA-1B®, Penn Century, Philadelphia, PA, USA) inserted into the trachea under visual control. No intra-operative deaths or delayed recovery (less than 2 min after inoculation) were observed. Birds were monitored at least twice daily and every signs of morbidity or mortalities were recorded.

All animal research procedures were approved by the regional ethics committee for animal experimentation at the veterinary college of Alfort.

\subsection{Sample collection}

Targeted organs of the respiratory tract from all dead and euthanized animals were removed aseptically under laminar flow hood. The trachea and left thoracic air sac were sampled with a sterile swab. The left lungs were collected for histology and the right lungs were used for fungal culture, real time PCR and galactomannan dosage.

\subsection{Histological evaluation}

Tissues collected for histology were immediately fixed in $10 \%$ neutral buffered formalin and dehydrated. Paraffin wax-embedded specimens were sectioned at $4 \mu \mathrm{m}$ and stained with hematoxylin-eosin-saffron (HES) to evaluate the inflammatory response. Periodic acid Schiff (PAS) and Grocott-Gomori methenamine-silver stains (MS) were used to visualize Aspergillus in lesions. A blind reading of 1 up to 10 slides per selected turkey of each group was conducted to evaluate the intensity of microscopic lesions. 
2.7. Fungal culture of respiratory organs

The right lungs were weighed and grinded with a homogenizer of tissue (Bio-Gen PRO200 Homogenizer, Pro Scientific, Oxford CT, USA) in $6 \mathrm{~mL}$ of PBS containing $0.01 \%$ (vol/vol)

Tween $20.100 \mu \mathrm{L}$ of the primary lung homogenates were immediately used for fungal culture, the rest was frozen at $-20^{\circ} \mathrm{C}$ until use.

Dilutions of primary lung homogenates and swabs of trachea and air sac were seeded on malt chloramphenicol agar plates and incubated at $37^{\circ} \mathrm{C}$. The count of A. fumigatus colonies (CFU) were assessed 1 and 2 days after plate inoculation.

\subsection{Assessment of conidial equivalent by real time PCR}

The primary lung homogenates were subjected to a secondary homogenization step. $500 \mu \mathrm{L}$ of lung homogenate was transferred to a sterile $2 \mathrm{~mL}$ screw-cap centrifuge tube containing $0.5 \mathrm{~mm}$-diameter glass beads (Biospec, Bartlesville, USA) and placed in a MagNa Lyser homogenizer (Roche, Meylan, France) for 3 bursts of $30 \mathrm{~s}$ at $3500 \mathrm{rpm}$ with incubation on ice between bursts. The secondary lung homogenate was collected by centrifugation at $800 \mathrm{~g}$ for $5 \mathrm{~min}$ at $4^{\circ} \mathrm{C}$ and $100 \mu \mathrm{L}$ of this homogenate was directly used for DNA extraction with the QIAamp DNA Mini Kit (Qiagen, Courtabeouf, France) according to the manufacturer's directions. Fungal DNA and secondary homogenate were stored at $-20^{\circ} \mathrm{C}$ until use. Realtime TaqMan PCR quantified the amount of A. fumigatus cell nuclei by targeting the singlecopy $F K S$ gene and the multi-copy gene encoding the $28 \mathrm{~S}$ rRNA. The sequences of the $F K S$ primers were previously reported [23]: sense amplification primer, AFKS1 (5'-GCC TGG TAG TGA AGC TGA GCG T-3'), antisens amplification primer, AFKS2 (5' -CGG TGA ATG TAG GCA TGT TGT CC-3') and the hybridization $F K S$ probe (5'-6-FAM-TCA CTC TCT ACC CCC ATG CCC GAG CC-BHQ1-3'). The 28S primers were reported by Challier et al. [24]: 28S-466 (5'-CTC GGA ATG TAT CAC CTC TCG G-3') and 28S-533 (5'-TCC 
TCG GTC CAG GCA GG-3') and the hybridization 28S probe (5'-6-FAM-TGT CTT ATA GCC GAG GGT GCA ATG CG- TAMRA-3’').

A standard curve was established with DNA samples extracted from uninfected lungs of turkeys inoculated with different doses of A. fumigatus conidia (from $10^{2}$ to $10^{8}$ conidia) and allowed us to quantify the Aspergillus DNA in the organs by calculating conidial equivalents (CE) [15]. DNA samples were analysed in duplicate by using the LightCycler® 480 detection system (Roche, Meylan, France). The reaction mixture consisted in $5 \mu \mathrm{L}$ of DNA, 900 or $500 \mathrm{nM}$ sense and antisens primers from $F K S$ or $28 \mathrm{~S}$ targets, $200 \mathrm{nM} F K S$ probe or $250 \mathrm{nM} 28 \mathrm{~S}$ probe and LightCycler® 480 Probes Master 2x (Roche, Meylan, France). All assays were run under the following conditions: $50^{\circ} \mathrm{C}$ for $2 \mathrm{~min}, 95^{\circ} \mathrm{C}$ for $10 \mathrm{~min}$, then 50 cycles of $15 \mathrm{~s}$ at $95^{\circ} \mathrm{C}$ and $1 \mathrm{~min}$ at $65^{\circ} \mathrm{C}$ or $60^{\circ} \mathrm{C}$ respectively for $F K S$ or $28 \mathrm{~S}$ primers.

\subsection{Galactomannan (GM) quantity assessment}

$500 \mu \mathrm{L}$ of primary lung homogenates were centrifuged at 2,300 $\mathrm{g}$ for $5 \mathrm{~min}$ and the supernatant was placed into a fresh tube. Lung supernatants $(300 \mu \mathrm{l})$ were used for GM determination with the Platelia GM-EIA kits (Bio-Rad Laboratories) according to the manufacturer's directions. The minimum and maximum thresholds of DO detected by the ELISA reader (Biorad PR1300) ranged between 0 and 3 Abs. A 1:100 dilution of lung homogenates was realized for all groups and a 1:1000 dilution for the Inoc8 group in order to avoid the saturation of the signal detected in some crude lung homogenates.

\subsection{Genotyping of Aspergillus fumigatus isolates}

A subset of strains recovered either from the environment or from internal organs was collected and genotyped by Multi Locus VNTR Analysis [25]. 
2.11. Statistical analyses

Statistical analyses were performed with the JMP 10.0 software. A log rank t test has been used to analyse the survival of birds and to evaluate the effect of the inoculum dose on mortality. A nonparametric one-way analysis of variance, Wilcoxon test, linear regression models and Spearman's rank correlation were performed for multiple comparisons and to evaluate the relationship between the different biomarkers $\left(\log _{10} \mathrm{CFU} / \mathrm{g}\right.$ of lung, $\log _{10} \mathrm{CE} / \mathrm{g}$ of lung and the GM index) and the inoculum doses.

\section{Results}

\subsection{Body weight, clinical signs and mortality}

A dose dependent response was observed concerning weight gain evolution when inoculated turkeys were compared to non-challenged birds in both sets. If birds from Inoc5 and Inoc6 were not affected by Aspergillus challenge, growth average of birds exposed to higher concentrations (Inoc7 and Inoc8) decreased significantly as soon as 2 days post inoculation (figure 1).

No clinical signs or mortality were observed in non-challenged turkeys and Inoc5. The clinical signs observed in turkeys of Inoc6, Inoc7 and Inoc8 included lethargy, ruffled feathers, progressive weight loss and respiratory distress such as open-mouthed breathing leading to lateral recumbency in severe cases. Two birds out of 9 in Inoc6 exhibited clinical signs 6 days after inoculation, but did not die before the end of the experiment. Eight turkeys out of 10 in Inoc 7 presented clinical signs, which appeared between 3 and 6 days after inoculation. Among them, only 3 animals died at day 5. In set B, an acute respiratory distress was registered between 1 and 3 days post inoculation of A. fumigatus in all turkeys of Inoc8 $(n=10)$, which died 24 to 48 h later (figure 2$)$. 
3.2. Macroscopic and microscopic lesions

230 No macroscopic lesions were observed on non-challenged and Inoc5 birds. An increasing proportion of turkeys from Inoc6 (5/10), Inoc7 (8/10) and Inoc8 (10/10) exhibited few granulomas on thoracic air sacs and a densification of variable proportions of the pulmonary parenchyma. Histological lesions associated with Aspergillus development, mostly represented by multifocal either isolated or coalescing granulomas with necrotic centers, and occurrence of multinucleated giant cells (figure 3) were confirmed in lungs of Inoc6, Inoc7 and Inoc8 turkeys but were absent in Inoc5 or non-challenged birds. Percentages of altered parenchyma varying from 30 to $80 \%$ have been estimated on lungs preparation coming from different birds belonging to Inoc7 and Inoc8. However, the hyphae burden seemed to be more important in the latter group.

3.3. Fungal cultures from trachea, air sacs and lungs

The lower limit of the assay is approximately $300 \mathrm{CFU} / \mathrm{g}$ of lung (10 CFU/plate). All nonchallenged turkeys exhibited negative cultures. Semi-quantitative numerations from trachea and air sacs showed variable levels of contamination immediately after inoculation in all challenged groups. However no Aspergillus could be recovered from both organs of all birds of Inoc5, Inoc6 and Inoc7 that were euthanized between 5 and 8 days post challenge. For Inoc8, a high level of contamination (>100 CFU/plate) was still present in air sacs till day 4, but not in trachea even in turkeys which died spontaneously 2 days after inoculation. Positive counts ranging from 3.28 up to $9.75 \log _{10} \mathrm{CFU} / \mathrm{g}$ have been registered on lungs of 250 all turkeys sacrificed immediately after the inoculation. In the remaining birds, the proportion of positive samples and the mean of $\log \mathrm{CFU} / \mathrm{g}$ of lung established on those 
13 turkeys (Inoc7 and Inoc8) that died spontaneously between 2 and 5 days pi was $5.47 \pm$ 0,41 .

255

3.4. Assessment of conidial equivalent by real time PCR

The sensitivity of real time PCR on 28S target was higher than on FKS gene with limits of detection varying from $5 \mathrm{CE}$ (corresponding to $10^{3} \mathrm{CE} / \mathrm{g}$ of lung) to $250 \mathrm{CE}$ per reaction $(5 \mathrm{x}$ $10^{4} \mathrm{CE} / \mathrm{g}$ of lung) respectively. Immediately after experimental infection, qPCR signals remained undetectable for the 2 birds of Inoc5, one turkey of Inoc6 but highly positive for the others (Inoc7 and 8). At day 8, no amplification at all was detected with both targets for Inoc5 and only $3 / 9$ samples from Inoc6 were detected with the $28 \mathrm{~S}$ target. The numbers of

CE/g established of lung detected with $F K S$ target and 28S target were positively correlated $\left(\log _{10} \mathrm{CE}_{\mathrm{FKS}} / \mathrm{g}\right.$ of lung $=-2.92+1.34 * \log _{10} \mathrm{CE}_{28 \mathrm{~S}} / \mathrm{g}$ of lung $)$ when assessed on the $17 \mathrm{DNA}$ samples (7/10 Inoc7 and 10/10 Inoc8) exhibiting amplification with both DNA targets (data not shown). Similarly to fungal numerations both proportions of positive amplifications and means of $\log \mathrm{CE}_{28 \mathrm{~S}} / \mathrm{g}$ of lung in positive results appeared to be correlated to the inoculum size (table 1).

GM index obtained on crude lung homogenates presented huge variations from 0.5 to 6 (signal saturation) in all groups including non-challenged birds. GM index repartition after 1:100 dilution (figure 4) was quite similar in control, Inoc5 and Inoc6 birds ( $\mathrm{p}=0.0084)$. The highest variation of GM index values was observed in Inoc7 (mean GM index $=3.99 \pm$ 0.90). All turkeys that died spontaneously (including all Inoc8) exhibited index higher than 5. Interestingly, GM index of all birds immediately euthanized after inoculation were lower than 0.35 . 
The number of $\log _{10} \mathrm{CE} 28 \mathrm{~S} / \mathrm{g}$ of lung was strongly correlated to the number of $\log _{10} \mathrm{CFU} / \mathrm{g}$ of lung $(\mathrm{p}<0.0001)$ when the data of all groups were included (figure 5) but not in each groups considered separately. Both values increased significantly with inoculum size $(\mathrm{p}<0.0001)$. As featured by the regression line, the sensitivity threshold of CE values was almost 10 fold higher than CFU counts in lung samples $\left(\log _{10} \mathrm{CE} 28 \mathrm{~S} / \mathrm{g}\right.$ of lung $=1.30 * \log _{10}$ CFU/g of lung). Birds, which died quickly, have constantly more CFU and CE in lungs than the other birds. GM index applied to diluted lung homogenates (1:1000) of Inoc8 was significantly correlated to $\log _{10} \mathrm{CE} / \mathrm{g}(\mathrm{p}=0.0172)$ but not with $\log _{10} \mathrm{CFU} / \mathrm{g}(\mathrm{p}=0.526)$ (figure $6)$.

\subsection{Environmental contamination}

Negligible levels of contamination by molds (Aspergillus spp. and Mucorales) have been detected in the litter or in the room's atmosphere prior to the installation of the birds or after inoculation (controls at day 1 and day 8). Feedstuffs remained constantly culture-negative.

Six A. fumigatus isolates collected from the animals and the environment presented the same MLVA genotype as that of the reference strain (CBS 144.89) used for the experimental infection. 


\subsection{Experimental model}

304

305

306

307

Many experimental aspergillosis have already been published using different avian models and testing various bird species, age at inoculation, challenge routes and even the Aspergillus species [1]. Both field data [2] and experimental results [26] clearly demonstrate a higher susceptibility of turkeys and quails to A. fumigatus infection when compared to chickens, but also intraspecific differences in host responses [4, 27] highlighting the necessity to establish models on relevant species or lineages reared for meat or egg production. Different routes of Aspergillus inoculation have been tested in turkeys including intrapulmonary [28], intra-air-sac administration [11, 21] and finally aerosolization in confined plastic chambers [3]. With the exception of the latter method, which is difficult to standardize [4], the intratracheal inoculation, which has not been yet tested in turkeys, is the closest to the natural way of infection and only bypasses the upper airways and their putative defenses [1]. We propose a relevant model of acute aspergillosis in one-week-old turkeys following intratracheal nebulization of Aspergillus fumigatus conidia with a MicroSprayer® aerosolizer. The use of this device has already been validated in immunosuppressed 6-8 weeks-old Sprague-Dawley rats [16] and immunocompetent Japanese quail [7] to administrate precisely calibrated inoculum of Aspergillus fumigatus in the respiratory tract. The evaluation of the impact of four inoculum concentrations $\left(10^{5}\right.$ to $10^{8}$ conidia per turkey) allowed us to record mortality following a dose response with values of $0,0,30$ and $100 \%$ respectively. Global parameters such as the evolution of body-weight, the occurrence and severity of clinical signs and the chronology of deaths appeared strictly in concordance with the concentration of conidia administered. Lower mortality rates (respectively 16, 55, 56 and $20 \%$ ) have been observed in turkeys treated with $10^{8}$ conidia in the air sac [21] or submitted to dry aerosol delivering doses of $5.10^{9}$ spores per animal [3] or in quails receiving $1.510^{6}$ 
327 [9] conidia intratracheally. A similar protocol ( $2.10^{7}$ conidia administered intratracheally)

328 applied to the same species resulted in 55\% [29] and 100\% mortality [8]. As mentioned by

329 Goetting et al. [7] who compared two modalities of inoculation, the nebulization by

330 Microsprayer® should not be considered to be strictly equivalent to a "classical"

331 intratracheal inoculation using an intravenous catheter after removing the stylet. Higher

332 mortality ( $88 \%$ versus $33 \%$ ) has indeed been observed after nebulization compared to the

333 "classical" method after the same inoculum size (4.7 $10^{7}$ spores). This could be attributed to

334 differences in dispersion parameters (velocity, dropplet size) through the respiratory tract.

335 Similarly to [7], the macroscopic lesions registered in our model were strictly limited to the

336 respiratory apparatus and particularly marked in lungs but much more rarely on caudal air

337 sacs. Extensive parenchyma necrotic damage consecutive with the presence of multifocal

typical granulomas $[11,13]$ were seen in the present experiment in all birds of Inoc8 but also in some animals belonging to Inoc7, which have been euthanized at the end of our

experiment. We cannot therefore exclude the possibility of delayed mortality (up to 50\%) in the latter group if the experiment took place over a longer post-inoculation period [3].

\subsection{Biomarker evaluation}

The relevance of three putative biomarkers (CFU, galactomannan and real-time PCR) has been concomitantly assessed on lung homogenates for the first time in turkeys. In contrast with already published models in the immunosuppressed rat [15] or Guinea pig [17, 18], our primary purpose was to study the kinetics of fungal development in several target organs but to compare the effect of variable inoculum sizes on these parameters and, in addition, we tried to establish potential correlations to characterize fungal burden states in the lung since it was the sole organ to present extensive macroscopic lesions in our model. 
Semi-quantitative culture-based methods used to evaluate the Aspergillus load in organs are easy to perform and common but only detect viable organisms. Furthermore, temporal studies in rodents suggest that these tools may not be accurate when monitoring the development of multicellular filamentous fungi particularly in parenchyma submitted to mechanical homogenization [30] leading to possible underestimation [31, 32]. Except for some air sacs samples obtained from turkeys, which died in the first four days following inoculation, no fungus could be detected on the trachea or air sac swabs. This may be explained by fungal clearance, or low sensitivity of the method used on these organs. Although not strictly comparable because the interval post-inoculation differed between dead and euthanized birds, the mean lung numerations, the proportion and the homogeneity of positive results seemed clearly to increase with the inoculum dose. All dead animals presented at least $5.10^{5}$ spores/g of lung tissue which was estimated by Richard et al. [3] as the dose necessary to kill about $50 \%$ of 3-week-old turkeys exposed to an aerosol of Aspergillus conidia which appeared to be a clear threshold in younger birds unable to mount an effective innate response $[4,11,13]$ in the critical phase of the first days following experimental infection. The detection of viable Aspergillus was possible in a high proportion of apparently healthy turkeys, which were euthanized at the end of the experiment, but may persist to least 8 weeks post-exposure [3]. However, fungal load in surviving birds as assessed by culture seemed to decrease post-inoculation as in other avian [4, 9] or mammal models $[18,19]$.

The enzyme immune assay of galactomannan, a specific cell-wall component of fungi is relatively easy to perform and is likely to reflect the progressive increase in mycelium load by tip extension of hyphae [31]. The availability of commercial tests has allowed this polysaccharide component to be measured as a valuable biomarker in the sera of different bird species suffering from aspergillosis [20]. It has been used in field studies on 
commercial broiler chickens and turkeys [22], but also in mammals [16, 32] and turkeys

[21] experimental models. As a potential diagnosis tool, contrasting results have been

obtained due to variable sensitivity or specificity depending on the species tested. Additional difficulties exist in the definition of an appropriate cut-off value and the stage of the disease [20] but also to the ubiquitous presence of galactomannan in the poultry environment leading to false positives [1]. This last point may partially explain the positive GM values obtained from crude lung homogenates of control birds housed in a clean but not sterile environment. This necessitates work on dilutions to evaluate this parameter. The GM index on 1:100 dilutions were very low in turkeys submitted to the two lowest doses as for nonexposed birds but were much more variable in Inoc7. This may reflect individual differences in host-response efficiency. The high GM index systematically seen in turkeys, which died spontaneously in contrast to almost undetectable values in birds euthanized immediately after receiving variable doses of non-germinated conidia, is consistent with the relationship between the index value and the progression of the fungus [17, 31]. Furthermore, GM index correlated with the extent of infection as demonstrated by histopathological examination [18].

A real-time quantitative PCR assay has been developed recently in rodent models to measure the fungal load and its progression in different organs in order to evaluate the efficacy of antifungal therapy $[15,17,18,19]$. This molecular tool allows the detection of both conidia and hyphae and can be very sensitive and specific but like the GM enzyme immunoassay, it does not indicate viability [31]. Gene targets included a monocopy gene like FKS1 [18] or multicopy genes, in particular the ribosomal DNA genes [15] and internal transcribed spacer. The choice of multicopy genes alone or in the frame of a nested PCR [17] to improve sensitivity may not be ideal for standardization of the method [32]. To our knowledge, qPCR in avian lungs was evaluated for the first time in this study. The real-time 
PCR signal from a sample of infected tissue was compared with a standard curve composed

402 of conidia added to naïve pulmonary parenchyma and we chose to express the fungal

403

404

405

406

407

408

409

410

411

412

413

414

415

416

417

418

419

420

421

422

423

424

425

biomass in terms of CE [15]. The performances of the monocopy $F K S$ gene and the

multicopy 28SrDNA amplification in turkey lungs proved to be equivalent although the

sensitivity of the latter PCR was logically about $1 \log _{10} \mathrm{CE} / \mathrm{g}$ lower. Mathematical

extrapolation predicted that the detection limit of this assay was approximately $10^{3} \mathrm{CE} / \mathrm{g}$ of lung, which is quite similar to $2.10^{3} \mathrm{CE} / \mathrm{g}$ (18S) estimated in an haematogenous mouse model of aspergillosis [15], but could further be improved with a nested qPCR [17]. As demonstrated by results on euthanized birds just after inoculation, the qPCR seemed to be less sensitive than CFU numerations, which may be explained by a low efficiency of extraction of conidia from tissue samples $[19,30]$. Although no signal could be amplified in Inoc5 and there was no sign of illness, both the proportion of positive samples and mean $\log _{10} \mathrm{CE} / \mathrm{g}$ tended to increase with the dose used. Finally, the onset of induced mortality coincided clearly with a peak in fungal burden in Inoc8 as observed in a mouse model [15]. A strong correlation between qPCR results and culture numerations was seen when positive data from all groups were included and the molecular method demonstrated a higher sensitivity. This relationship appears to be stronger when tissues contain hyphae rather than conidia [30]. When data from Inoc8 were compared with similar mammal models [15, 18], GM index and qPCR appeared to be more closely correlated with the rise of infection in comparison with CFU values which may partially be explained by the fact that the culturenumerations stagnates or decreases with time [19].

\subsection{Conclusion}

We have developed a repeatable and standardized model of acute and fatal primary pulmonary pneumonia in the young, immunocompetent turkey using an atomizer, which 
426 may be applicable to chemotherapy trials. Housing birds in groups in a controlled

427 environment enables field conditions of production to be mimicked and to evaluate the 428 treatment of aspergillosis by nebulized molecules such as azole compounds [2]. Besides

429 survival, still considered the most reliable measure to assess antifungal therapy, the

430 combination of additional markers of fungal development may contribute to an earlier and

431 more precise detection of treatment efficiency in birds submitted to lethal or sub-lethal

432 challenges but also to the refinement and the reduction objectives of in vivo experimental

433 research $[31,32]$.

434

435 Acknowledgement

436 EM received a grant Conventions Industrielles de Formation par la Recherche (CIFRE)

437 from Elanco Company. We thank Professeur Andrew Ponter for the careful relecture of the 438 article. 
439 Table 1. Fungal burdens in lungs of inoculated turkeys measured by CFU and real time PCR $440 \quad(28 \mathrm{~S})$ assays.

\begin{tabular}{lcc}
\hline Groups & Mean $\log _{10} \mathrm{CFU} / \mathrm{g}$ of lung & Mean $\log _{10} \mathrm{CE}_{28 \mathrm{~s}} / \mathrm{g}$ of lung \\
\hline Inoc5 & BLD & BLD \\
Inoc6 & $2.84 \pm 0.30(5 / 9)^{\mathrm{b}}$ & $5.32 \pm 0.13(3 / 9)$ \\
Inoc7 & $4.34 \pm 0.29(7 / 10)$ & $5.73 \pm 0.14(7 / 10)$ \\
Inoc8 & $5.60 \pm 0.12(10 / 10)$ & $7.17 \pm 0.11(10 / 10)$ \\
\hline
\end{tabular}


443 Figure 1. Mean weight variations of inoculated birds (black line) compared to non-

444 challenged turkeys (grey line) from set A (solid line) or B (dashed line). Inoculum of $10^{5}$ $445(\triangleright), 10^{6}(\triangle), 10^{7}(\diamond)$ or $10^{8}(\bigcirc)$ Aspergillus fumigatus conidia.

446

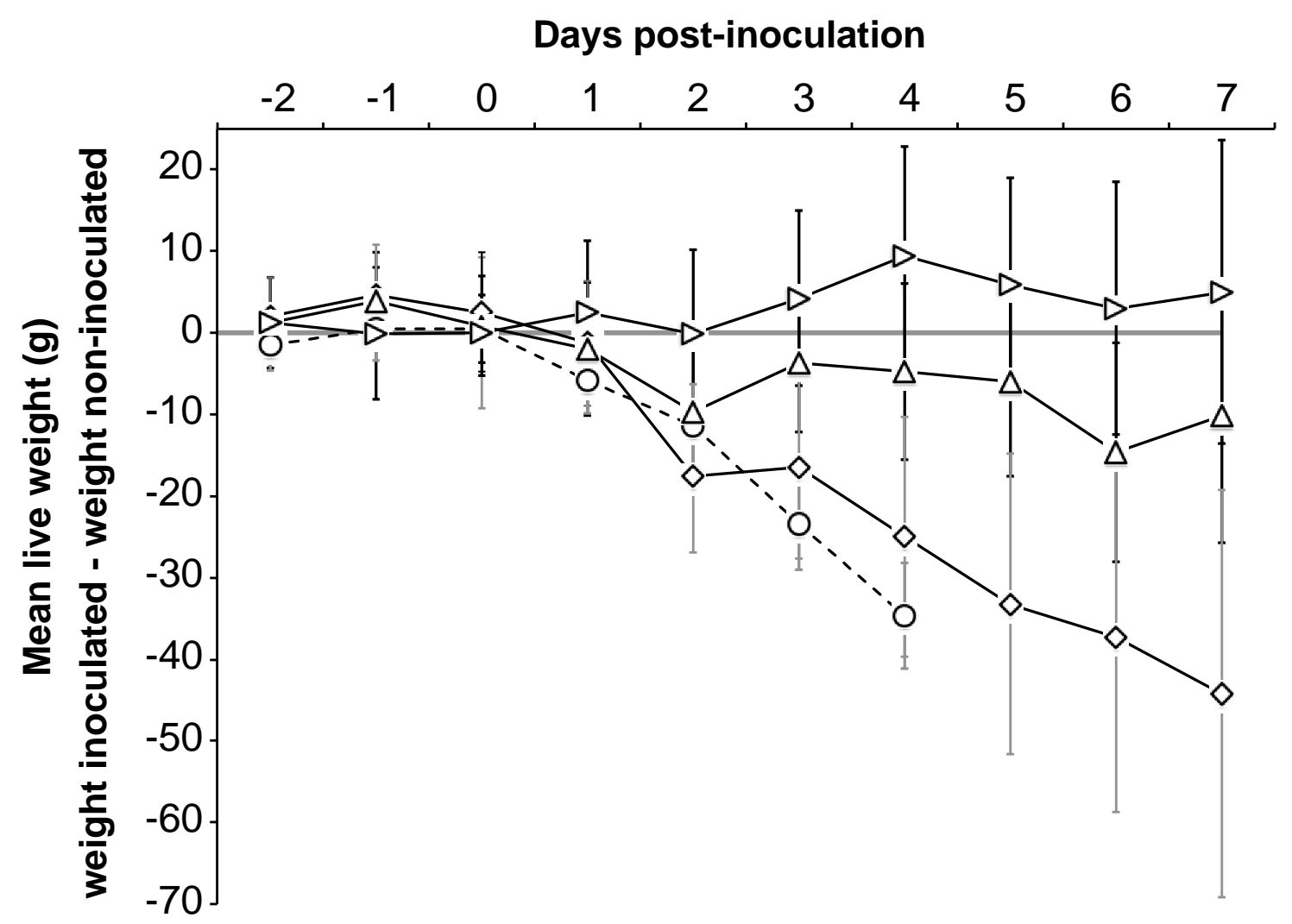

447 
Figure 2. Survival time of non-challenged (grey line) and inoculated (black line) turkeys

450 from set A (solid line) and B (dashed line) Inoculum of $10^{5}(\triangleright), 10^{6}(\triangle), 10^{7}(\diamond)$ or $10^{8}$ 451 (O) Aspergillus fumigatus conidia.

452

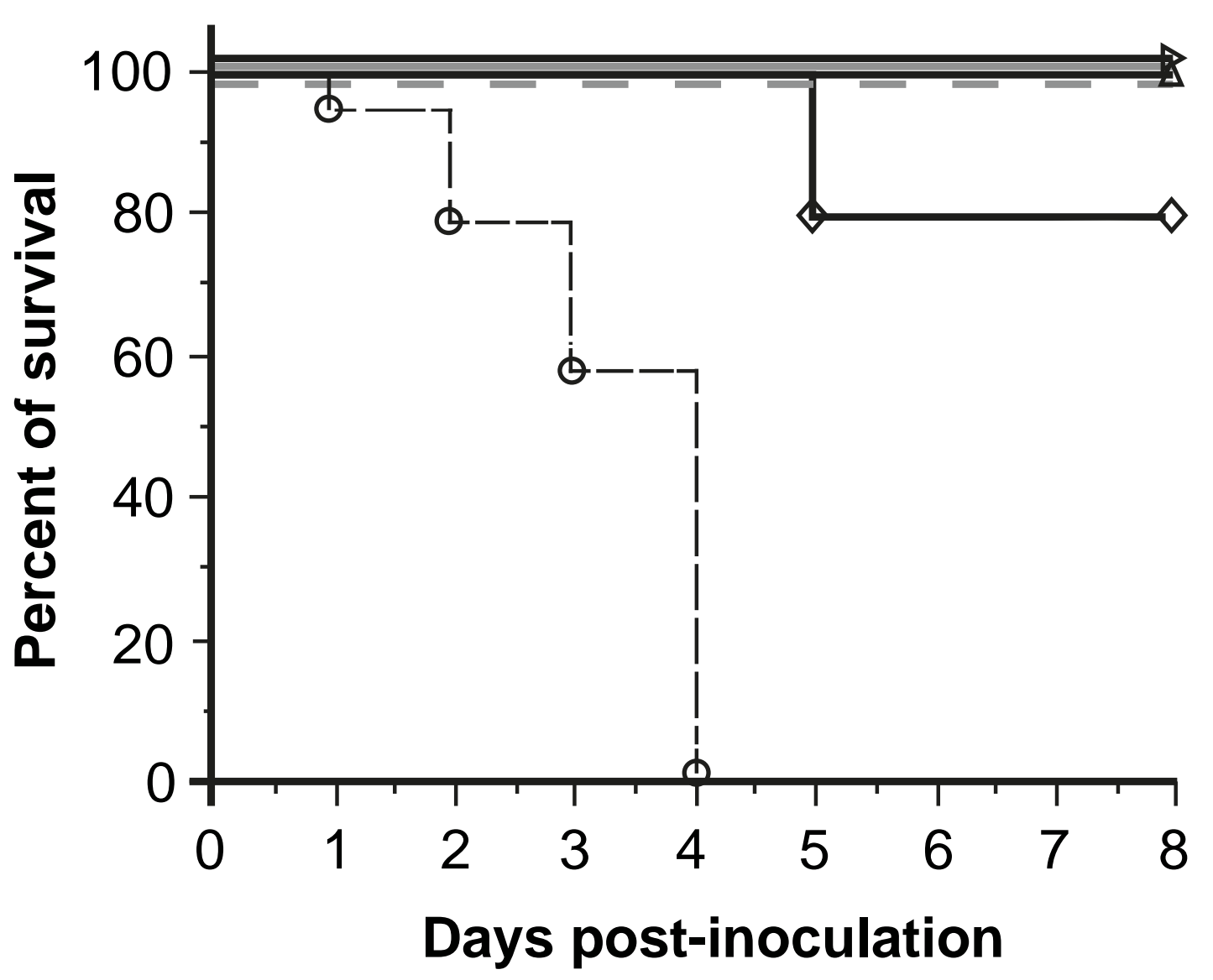


461 Figure 3. Histological sections of lungs from 2 turkeys (Inoc8). 3a (HES stain): Voluminous

462 granuloma with a necrotic centre. 3b, 3c (HES stain) and 3d (MS stain): Detailed of a

463 granuloma. A: necrosis; B: granuloma; C: red blood cells in capillary; D: giant cells with

464 multiple nuclei; E: Aspergillus hyphae.

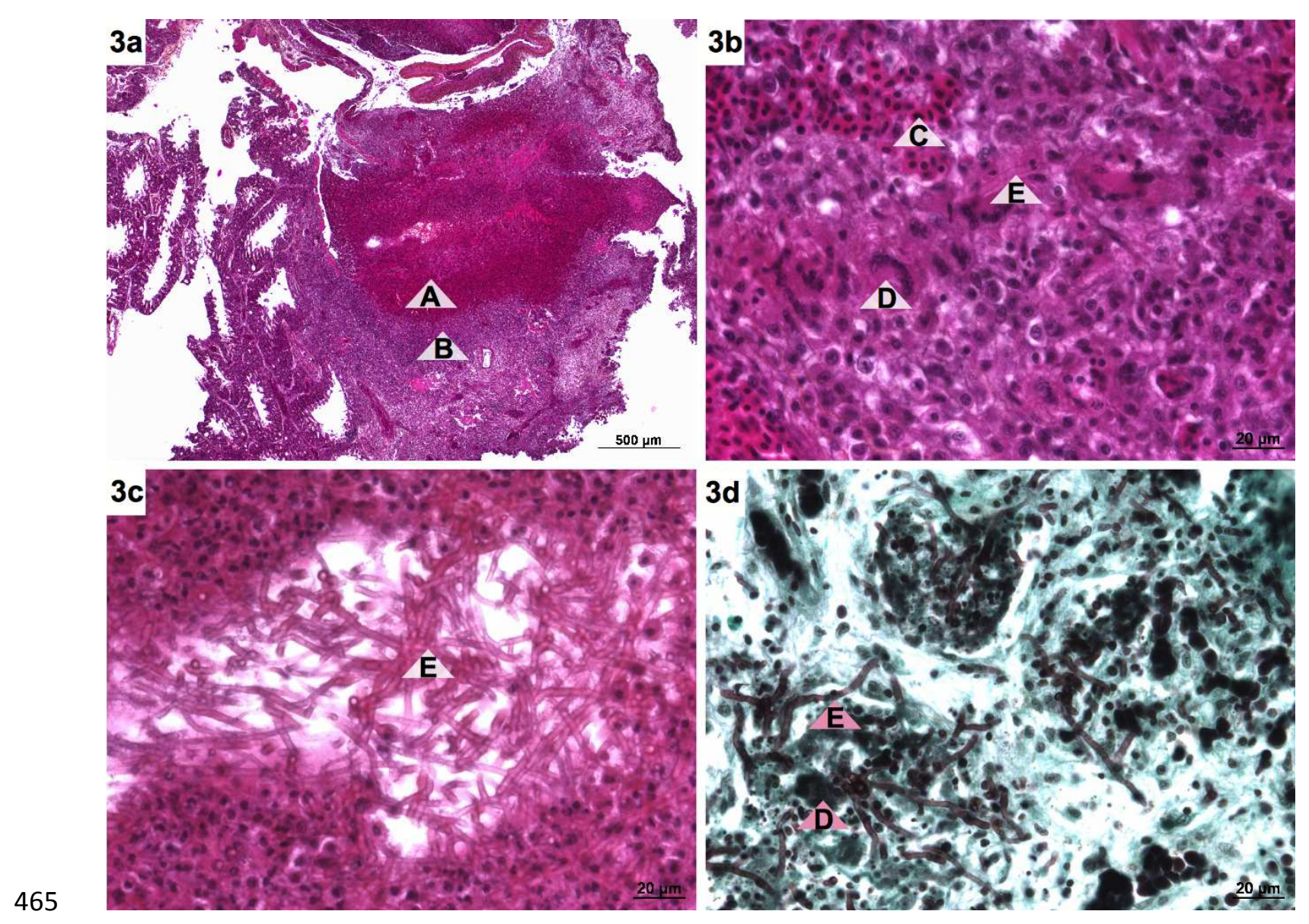


468 non challenged and inoculated groups.

469

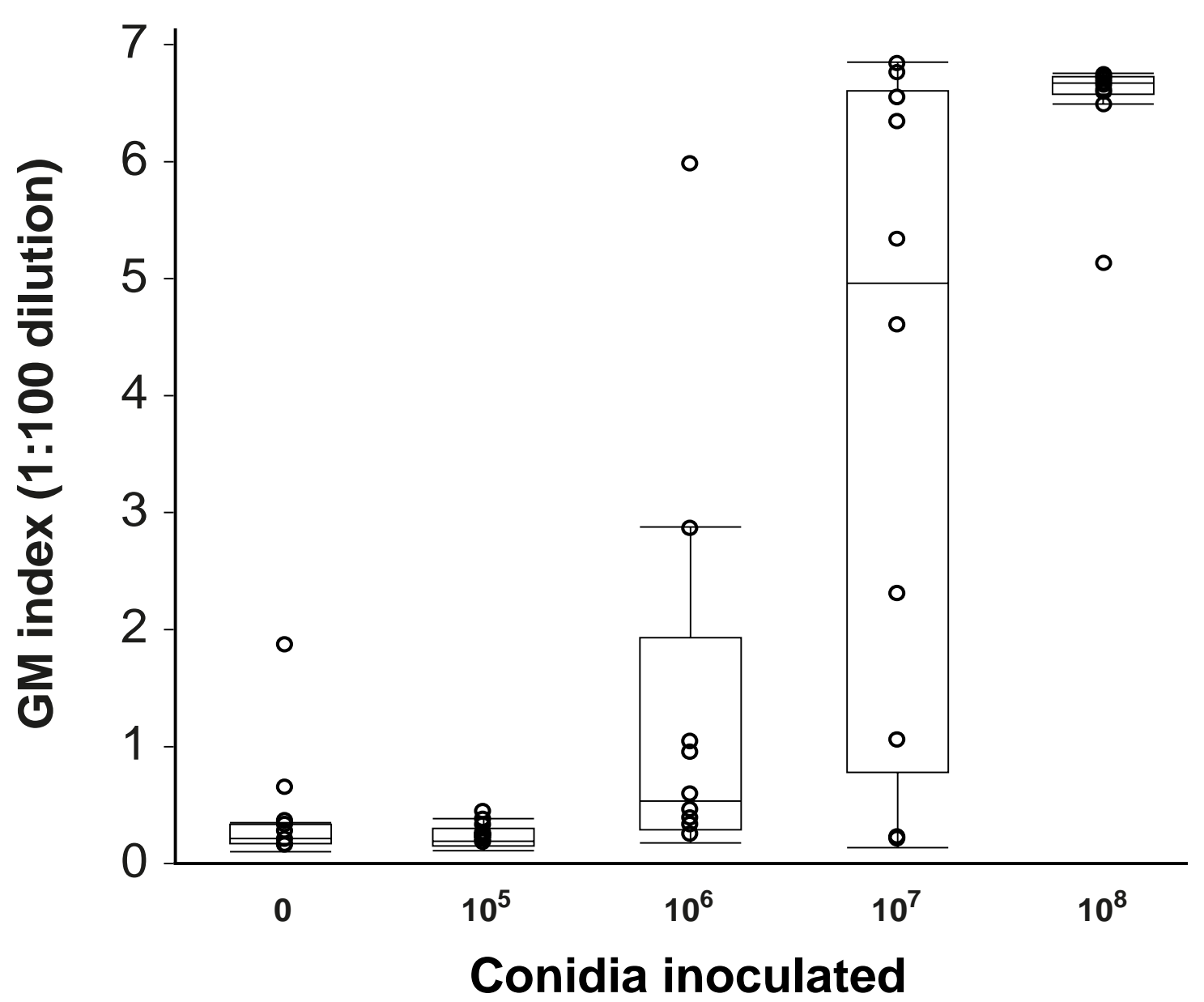


Figure 5. Relationship between CFU and CE $\left(\log _{10} \mathrm{CE} 28 \mathrm{~S} / \mathrm{g}\right.$ of lung $=1.30 * \log _{10} \mathrm{CFU} / \mathrm{g}$ of 481 lung) as shown by linear regression. Turkeys inoculated with $10^{5}(\triangleright), 10^{6}(\triangle), 10^{7}(\diamond)$ or $48210^{8}(\mathrm{O})$ conidia. *Significant correlation $(\mathrm{p}<0.0001)$ between results by the Spearman rank 483 correlation, $\mathrm{n}=21$.

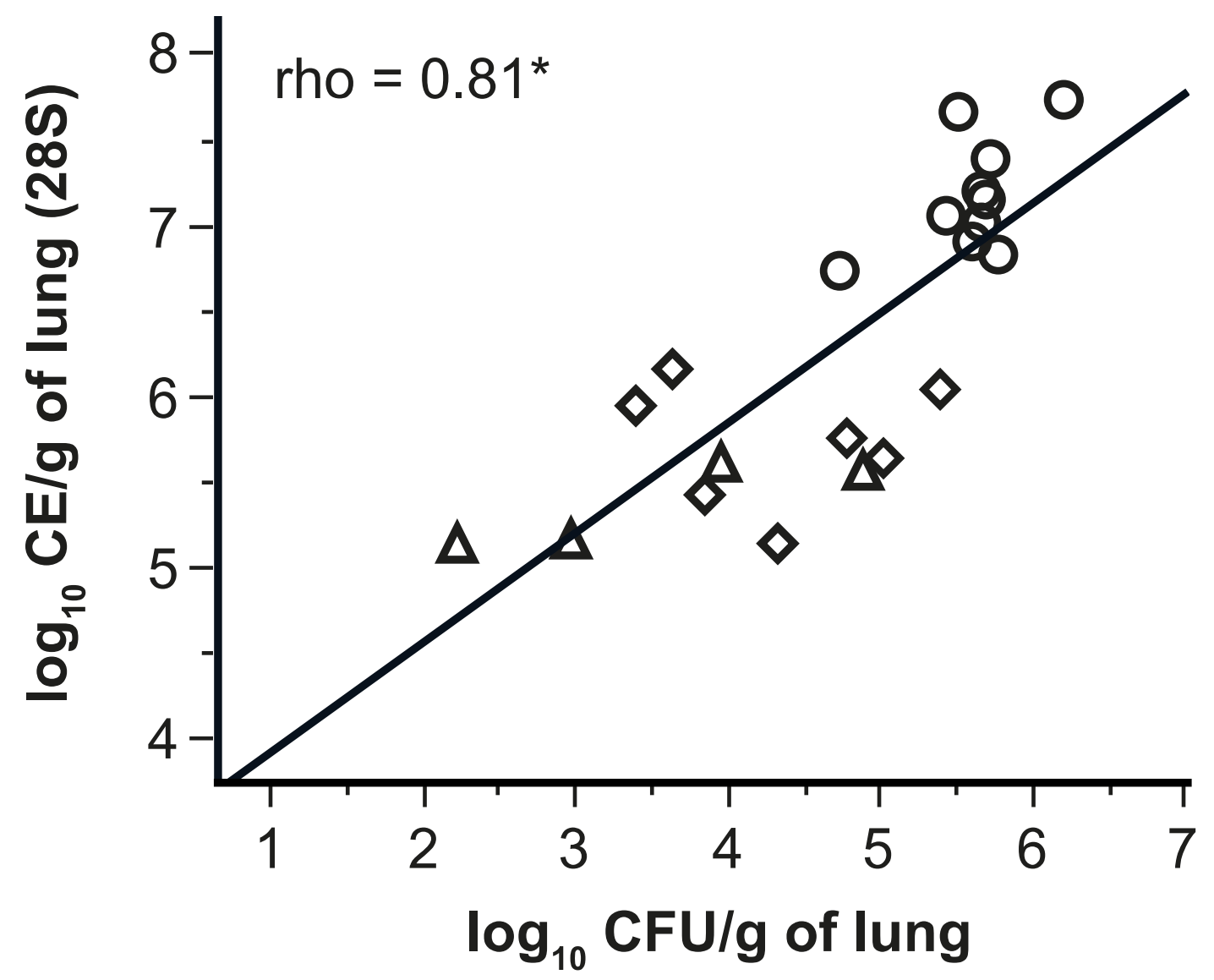


494 turkeys inoculated with $10^{8}$ conidia. *Significant correlation $(\mathrm{p}=0.0038)$ between 495 galactomannan index and CE by the Spearman rank correlation test, $\mathrm{n}=10$.
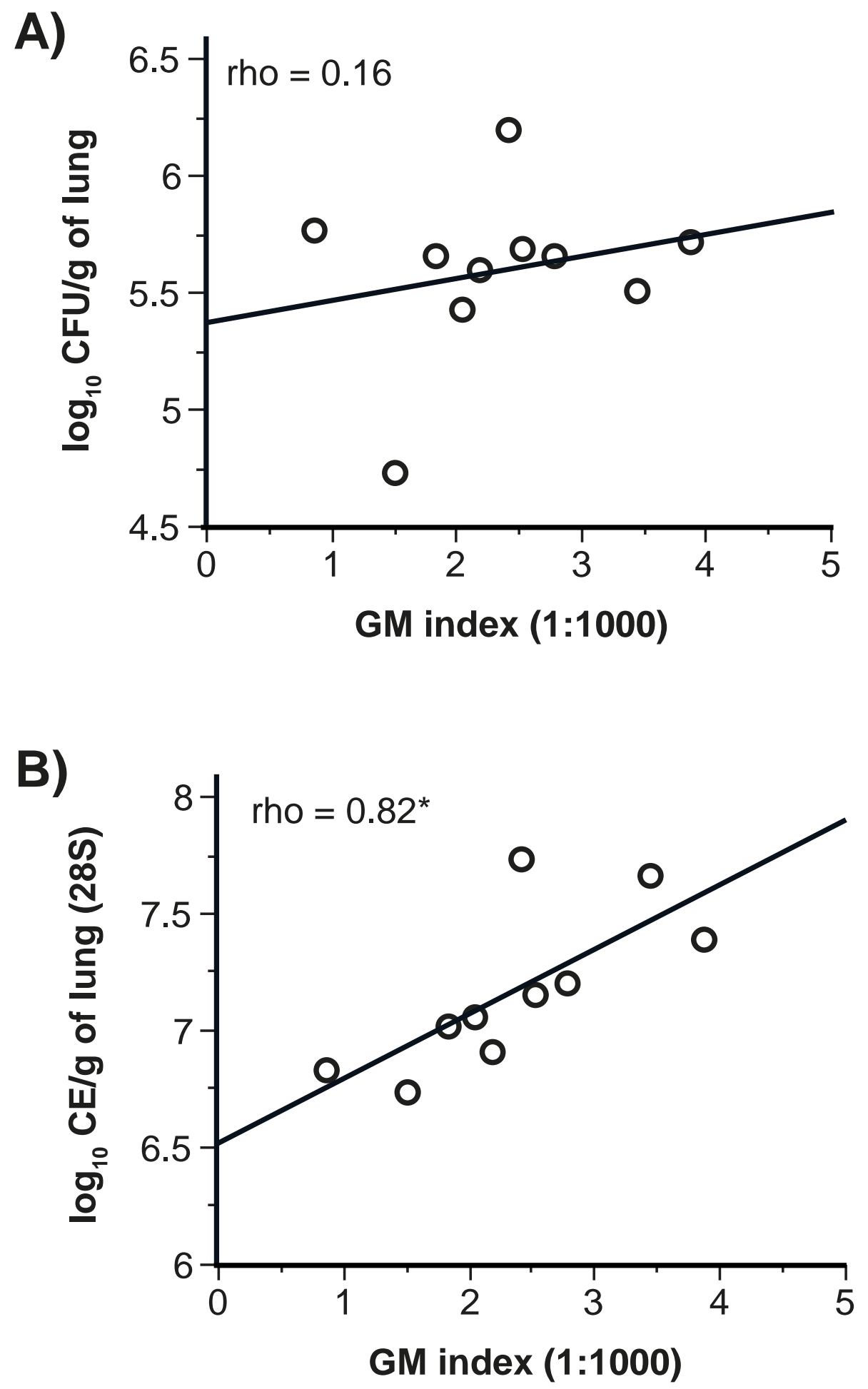


\section{BIBLIOGRAPHY}

500 [1] P. Arné, S. Thierry, D. Wang, M. Deville, G. Le Loc’h, A. Desoutter, F. Féménia, A. Nieguitsila, W. Huang, R. Chermette, J. Guillot, Aspergillus fumigatus in Poultry, Int. J. Microbiol. (2011) 2011:746356 doi:10.1155/2011/746356

503

504

505

506

507

508

509

510

511

512

513

514

515

516

517

518

519

520

521

[2] R.A. Kunkle, Aspergillosis, in: Y.M. Saif, H.J. Barnes, J.R. Glisson, et al. (Eds.), Diseases of Poultry, Iowa State University Press, Ames, Iowa, USA, 11th edition, 2003, pp. 883-895.

[3] J.L. Richard, R.C. Cutlip, J.R. Thurston, J. Songer, Response of turkey poults to aerosolized spores of Aspergillus fumigatus and aflatoxigenic and nonaflatoxigenic strains of Aspergillus flavus, Avian Dis. 25 (1) (1981) 53-67.

[4] S. Thierry, B. Durand, E. Melloul, J. Tafani, D. Ying, M. Deville, N. Cordonnier, R. Chermette, J. Guillot, P. Arné, Assessment of Aspergillus fumigatus pathogenicity in aerosol-challenged chickens (Gallus gallus) belonging to two lineages, Comparative Immunol. Microbiol. Infect. Dis. 36 (4) (2013) 379-385.

[5] R.A. Kunkle, R.B. Rimler, E.M. Steadham, Absence of protection against challenge with Aspergillus fumigatus by adoptive transfer of splenocytes from convalescent turkeys, Avian Dis. 43 (4) (1999) 678-684.

[6] A. Atasever, K.S. Gumussoy, Pathological, clinical and mycological findings in experimental aspergillosis infections of starlings, J. Vet. Med. A Physiol. Pathol. Clin. Med. 51 (1) (2004) 19-22.

[7] V. Goetting, K. Lee, L. Woods, K.V. Clemons, D. Stevens, L. Tell, Inflammatory marker profiles in an avian experimental model of aspergillosis, Med. Mycol. 51 (7) (2013) 696-703. 
522 [8] K.S. Gümüsşsoy, F. Uyanik, A. Atasever, Y. Cam, Experimental Aspergillus fumigatus infection in quails and results of treatment with itraconazole, J. Vet. Med. B Infect. Dis. Vet. Public Health 51 (1) (2004) 34-38.

[9] L.A. Tell, K.V. Clemons, Y. Kline, L. Woods, P.H. Kass, M. Martinez, D.A. Stevens, Efficacy of voriconazole in Japanese quail (Coturnix japonica) experimentally infected with Aspergillus fumigatus, Med. Mycol. 48 (2) (2010) 234-244.

[10] L. Van Waeyenberghe, D. Fischer, T. Coenye, R. Ducatelle, F. Haesebrouck, F. Pasmans, M. Lierz, A. Martel, Susceptibility of adult pigeons and hybrid falcons to experimental aspergillosis, Avian Pathol. 41 (6) (2012) 536-537.

[11] F. Féménia, J.J. Fontaine, S. Lair-Fulleringer, N. Berkova, D. Huet, N. Towanou, F. Rakotovao, O.I Granet, G. Le Loc'h, P. Arné, J. Guillot, Clinical, mycological and

[15] J.C. Bowman, G.K. Abruzzo, J.W. Anderson, A.M. Flattery, C.J. Gill, V.B. Pikounis,

[13] R.A. Kunkle, R.B. Rimler, Pathology of acute aspergillosis in turkeys, Avian Dis. 40 (4) (1996) 875-886.

[14] T.F. Patterson, Clinical utility and development of biomarkers in invasive aspergillosis, Trans. Am. Clin. Climatol. Assoc. 122 (2011) 174-183. D.M. Schmatz, P.A. Liberator, C.M. Douglas, Quantitative PCR assay to measure Aspergillus fumigatus burden in a murine model of disseminated aspergillosis: 
demonstration of efficacy of caspofungin acetate, Antimicrob. Agents Chemother. 45 (12) (2001) 3474-3481.

[16] J. Chandenier, S. Bernard, J. Montharu, E. Bailly, F. Fetissof, M. de Monte, G. Desoubeaux, P. Diot, D. Richard-Lenoble, The utility of a nebulised intra-tracheal rat model of invasive pulmonary aspergillosis, Mycoses 52 (3) (2009) 239-245.

[17] M. Lengerova, I. Kocmanova, Z. Racil, K. Hrncirova, S. Pospisilova, J. Mayer, L.K. Najvar, N.P. Wiederhold, W.R. Kirkpatrick, T.F. Patterson, Detection and measurement of fungal burden in a guinea pig model of invasive pulmonary aspergillosis by novel quantitative nested real-time PCR compared with

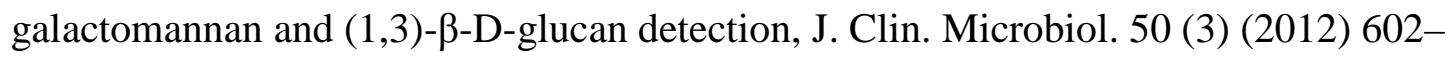
608.

[18] A.C. Vallor, W.R. Kirkpatrick, L.K. Najvar, R. Bocanegra, M.C. Kinney, A.W. Fothergill, M.L. Herrera, B.L. Wickes, J.R. Graybill, T.F. Patterson, Assessment of Aspergillus fumigatus burden in pulmonary tissue of guinea pigs by quantitative PCR, galactomannan enzyme immunoassay, and quantitative culture, Antimicrob. Agents Chemother. 52 (7) (2008) 2593-2598.

[19] Y. Zhao, S. Park, P. Warn, R. Shrief, E. Harrison, D. Perlin, Detection of Aspergillus fumigatus in a rat model of invasive pulmonary aspergillosis by real-time nucleic acid sequence-based amplification, J. Clin. Microbiol. 48 (4) (2010) 1378-1383.

[20] C. Cray, T. Watson, M. Rodriguez, K.L. Arheart, Application of galactomannan analysis and protein electrophoresis in the diagnosis of aspergillosis in avian species, J. Zoo Wildl. Med. 40 (1) (2009) 64-70.

[21] G. Le Loc’h, P. Arné, C. Bourgerol, E. Risi, J. Pericard, J. Quinton, S. Bretagne, J. Guillot, Detection of circulating serum galactomannan for the diagnosis of avian aspergillosis, $16^{\text {th }}$ Congress Intl. Soc. Hum. Anim. Mycol. (2006). 
[22] M. França, C. Cray, H.L. Shivaprasad, Serologic testing for aspergillosis in commercial broiler chickens and turkeys, Avian Dis. 56 (1) (2012) 160-164.

[23] C. Costa, D. Vidaud, M. Olivi, E. Bart-Delabesse, M. Vidaud, S. Bretagne, Development of two real-time quantitative TaqMan PCR assays to detect circulating Aspergillus fumigatus DNA in serum, J. Microbiol. Methods 44 (2001) 263-269.

[24] S. Challier, S. Boyer, E. Abachin, P. Berche, Development of a serum-based taqman real-time PCR assay for diagnosis of invasive aspergillosis, J. Clin. Microbiol. 42 (2) (2004) 844-846.

[25] S. Thierry, D. Wang, P. Arné, M. Deville, B. De Bruin, A. Nieguitsila, C. Pourcel, K. Laroucau, R. Chermette, W. Huang, F. Botterel, J. Guillot, Multiple-locus variablenumber tandem repeat analysis for molecular typing of Aspergillus fumigatus, BMC Microbiol. 10 (315) (2010) 1-8 doi:10.1186/1471-2180-10-315

[26] H.M. Ghori and S.A. Edgar, Comparative susceptibility of chickens, turkeys and Coturnix quail to aspergillosis, Poult. Sci. 52 (6) (1973) 2311-2315.

[27] R.A. Kunkle and R.E. Sacco, Susceptibility of convalescent turkeys to pulmonary aspergillosis, Avian Dis. 42 (4) (1998) 787-790.

[28] B. Perelman, B. Smith, D. Bronstein, A. Gur-Lavie, E.S. Kuttin, Use of azole compounds for the treatment of experimental aspergillosis in turkeys, Avian Pathol. 21 (4) (1992) 591-599.

[29] S.K. Chaudhary and J.R. Sadana, Experimental aspergillosis in Japanese quails (Coturnix coturnix japonica). Clinical signs and haematological changes, Mycopathologia 102 (3) (1988) 179-184. 
592 [30] D.C. Sheppard, K.A. Marr, D.N. Fredricks, L.Y. Chiang, T. Doedt, S.G. Filler,

593 Comparison of three methodologies for the determination of pulmonary fungal burden

594 in experimental murine aspergillosis, Clin. Microbiol. Infect. 12 (4) (2006) 376-380.

595 [31] K.V. Clemons, D.A. Stevens, Conventional or molecular measurement of Aspergillus $596 \quad$ load, Med. Mycol. 47 (Suppl 1) (2009) S132-S137.

597 [32] S. Seyedmousavi, W.J. Melchers, P.E. Verweij, J.W. Mouton, Assessment of efficacy 598 of antifungals in experimental models of invasive aspergillosis in an era of emerging 599 resistance: the value of real-time quantitative PCR, Curr. Opin. Pharmacol. 11 (5) $600 \quad$ (2011) 486-493. 